\title{
Hybrid
}

Revue des arts et médiations humaines

7 | 2021

Le réseau créatif des langu.ages

\section{Traduire une œuvre de littérature numérique en plusieurs langues : une étude de cas}

\section{Serge Bouchardon et Nohelia Meza}

Traducteur : Armelle Chrétien

\section{OpenEdition}

\section{Journals}

Édition électronique

URL : https://journals.openedition.org/hybrid/666

DOI : $10.4000 /$ hybrid. 666

ISSN : 2276-3538

Cet article est une traduction de :

Translating a work of digital literature into several languages: a case study - URL : https:// journals.openedition.org/hybrid/672 [en]

\section{Éditeur}

Presses universitaires de Vincennes

Référence électronique

Serge Bouchardon et Nohelia Meza, «Traduire une œuvre de littérature numérique en plusieurs langues : une étude de cas », Hybrid [En ligne], 7| 2021, mis en ligne le 08 avril 2021, consulté le 13 avril 2022. URL : http://journals.openedition.org/hybrid/666 ; DOI : https://doi.org/10.4000/hybrid.666

Ce document a été généré automatiquement le 13 avril 2022.

Revue Hybrid 


\title{
Traduire une œuvre de littérature numérique en plusieurs langues : une étude de cas
}

\author{
Serge Bouchardon et Nohelia Meza
}

Traduction : Armelle Chrétien

\section{Introduction}

1 Cet article propose un aperçu de différentes expériences relatives à la traduction du récit interactif Déprise ${ }^{1}$, avec pour but l'étude des différentes stratégies mises en œuvre dans le travail traductif. À cette fin, nous avons demandé aux traductrices et traducteurs (en anglais, italien, espagnol et portugais) leurs impressions sur ce processus: Valérie Bouchardon (Loss of Grasp, 2010), Giovanna di Rosario (Perdersi, 2011), Martha Asunción Alonso (Perderse, 2013) et Diogo Marques (Perda de controlo, 2016). Sur la base d'échanges écrits avec les traducteurs et les traductrices, nous aborderons ici les enjeux suivants: 1) Les modes de collaboration entre auteur et traducteur (stratégie de programmation entre auteur et traducteur; littératie numérique du traducteur et familiarité avec la littérature numérique; visibilité du traducteur) ; 2) L'importance de la traduction des dimensions propres à la littérature numérique (migration de code, formes et gestes sémiotiques, voix intégrées). Nous évoquerons également plusieurs axes de recherche futurs sur la traduction de littérature numérique (rôle de la traduction indirecte ${ }^{2}$, dimension culturelle des œuvres et la traduction comme mémoire réinventée). Enfin, conformément aux quatre dimensions de la traduction de littérature numérique définies par Manuel Portela, Søren Pold et María Mencía ${ }^{3}$ - 1) Translinguistique 2) Transcodage 3) Transmédialité 4) Transcréation - nous chercherons à déterminer si ces dimensions sont présentes dans l'expérience des traducteurs et des traductrices de Déprise. 


\section{Étude de cas : Déprise (2010)}

\section{Aspects généraux de l'œuvre numérique}

2 Déprise (ci-après DP) est un récit interactif inclus dans la trilogie Hyper-tensions, composée de trois œuvres: Déprise ${ }^{4}$, Opacité 5 et Détrace $^{6}$. DP fait partie de l'ELMCIP Anthology of European Electronic Literature ${ }^{7}$ et la version de l'œuvre en langue anglaise a remporté le New Media Writing Prize en 2011. DP est constitué de six scènes dépeignant les paysages mentaux d'un homme qui perd progressivement prise sur la vie. Dans la première scène, le protagoniste fait le point sur son existence - il traverse une période tumultueuse, une déprise; dans la scène 2 , il fait la rencontre de sa future femme lors d'un rendez-vous; dans la scène 3, il lit la double lettre de rupture/d'amour laissée par sa femme ; dans la scène 4 , il lit un texte poignant rédigé par son fils; dans la scène 5 , il est face à une confrontation avec lui-même, une déprise absolue; dans la scène 6, il décide d'affronter la situation pour reprendre sa vie en main. De scène en scène, le lecteur est mis au défi de déchiffrer la relation entre les imaginaires de l'interface numérique et sa propre personne. L'œuvre fut initialement programmée avec le logiciel Flash en 2010 avant de migrer vers JavaScript en 2018. Bien que le présent article se base sur les versions anglaise, italienne, espagnole et portugaise programmées avec Flash, on notera que les versions arabe, chinoise, allemande, hongroise, polonaise et russe, sorties en janvier 2020, ont uniquement été programmées en JavaScript ${ }^{8}$.

Fig. 1

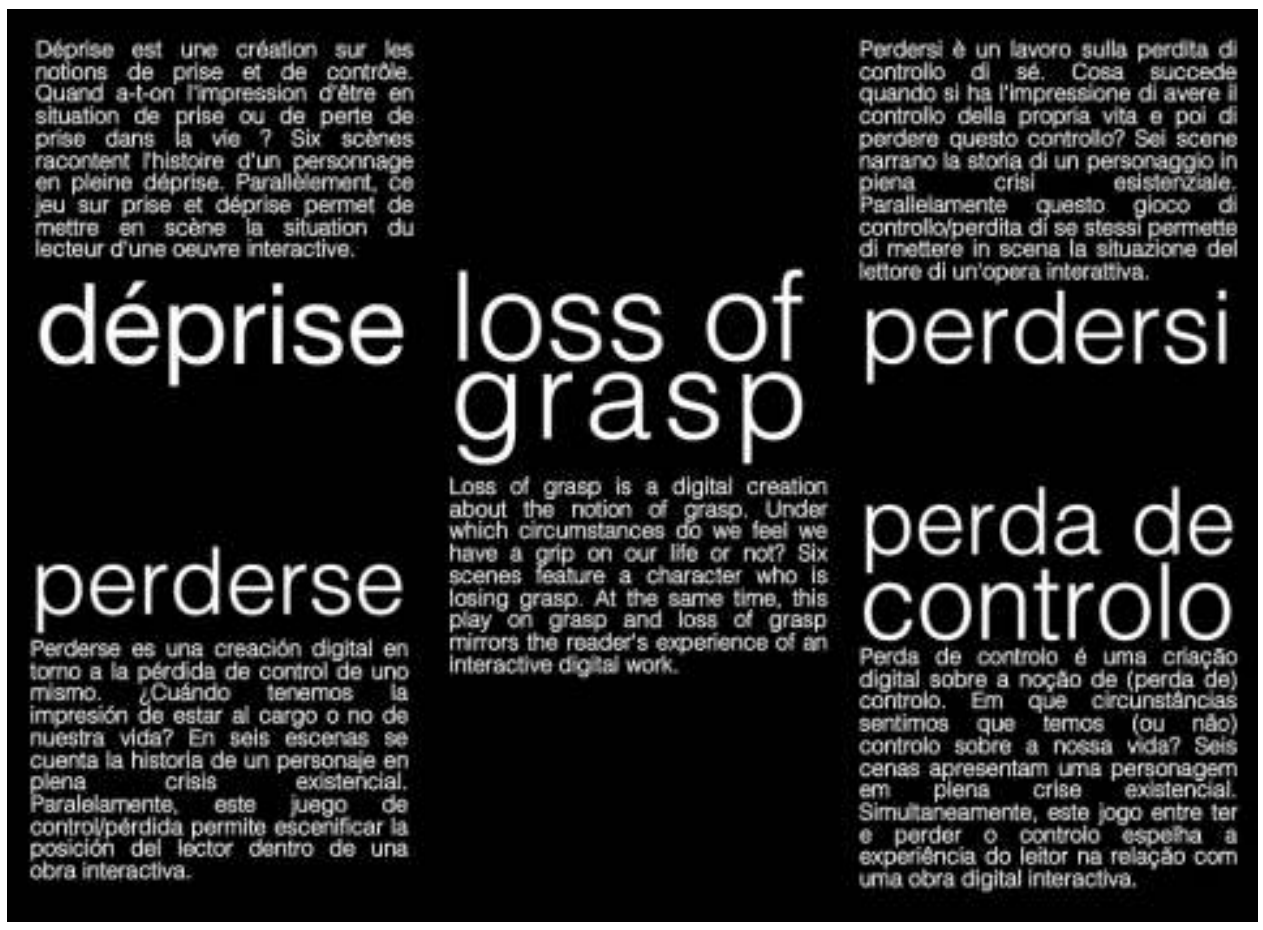

Page d'accueil de Déprise (version Flash). 


\section{Les entretiens}

3 Les premiers échanges et entretiens ont été réalisés la même année que les traductions. Ils ont concerné Valérie Bouchardon (Loss of Grasp, 2010), Giovanna di Rosario (Perdersi, 2011), Martha Asunción Alonso (Perderse, 2013) et Diogo Marques (Perda de controlo, 2016). Les traducteurs et les traductrices ont ensuite été recontactée's en 2018 et 2020. Toutes les communications se sont déroulées par mail, et les questionnaires étaient rédigés en français et en anglais. Les langues de communication entre l'auteur et les traducteurs et les traductrices étaient les suivantes: V. Bouchardon (français), di Rosario (français/anglais), Alonso (français), Marques (anglais). Le multilinguisme a ainsi d'office eu sa place au sein du processus. Diogo Marques, qui a travaillé sur la traduction portugaise, a notamment utilisé la version anglaise comme texte source et comparé certains aspects de sa traduction avec la version originale en français, de même qu'avec les versions espagnole et italienne, tout en communiquant avec l'auteur en anglais. Les échanges entre l'auteur et les traducteurs et les traductrices ont incontestablement conduit à un dialogue interculturel et multilingue.

\section{Différentes formes de collaboration}

\section{Entre texte et code}

4 En littérature numérique, il arrive que le code introduise une distance entre le traducteur et l'auteur. Le degré de collaboration entre auteur et traducteur aura un impact sur le travail de programmation du texte traduit en différentes langues. Pensons par exemple à l'importance de la manipulation sémiotique pour la recréation d'effets littéraires similaires dans les différentes versions d'une œuvre numérique. Dans le cas de $D P$, la plupart des traducteurs et des traductrices ont envoyé le texte linguistique à l'auteur sans prendre part à l'adaptation du code à chaque version.

$5 \mathrm{Au}$ cours de l'entretien mené auprès d'elle, V. Bouchardon a fait part de son impression : «J'ai préféré travailler à partir du fichier code. J'avais besoin de voir le contexte de codage pour mieux comprendre le processus à l'œuvre entre le code et ce qui apparaît à l'écran. » Cette remarque pose la question de l'opacité structurelle entre le code et les formes de restitution à l'écran. Di Rosario développe une réflexion similaire dans son approche des figures de rhétorique du texte et de leur reproduction :

Ma première préoccupation a toujours été de reproduire les «mouvements » du texte original dans ma traduction. Il ne s'agit pas uniquement d'un problème de langue, bien sûr, et si je pense à Déprise et à toute ses «figures de rhétorique », je me demande encore si je suis parvenue à toutes les rendre en italien. Mon objectif était de ne pas perdre le texte numérique "invisible », et je ne parle pas ici du code mais de ce que le code permet à l'écrivain ou au lecteur de faire.

6 Ces deux exemples mettent en lumière la spécificité de la littérature numérique quand il s'agit de définir les frontières entre code, auteur et traducteur. Comment la distance $d u$ code entre l'auteur et le traducteur affecte-t-elle la programmation de l'œuvre numérique dans ses différentes versions? Après les entretiens, nous avons remarqué qu'une fois le texte linguistique envoyé à l'auteur, programmé dans sa nouvelle version et publié dans une version bêta entre l'auteur et le traducteur, il n'était pas rare que les traducteurs et les traductrices demandent à apporter des modifications après avoir lu et interagi avec leur version. Nous avons observé que le temps de lecture (à l'écran), 
tout comme les questions d'interaction et de manipulation, jouaient un rôle essentiel dans le processus de traduction à partir des versions tests.

\section{Littératie numérique du traducteur et familiarité avec la littérature numérique}

7 La première étude critique de la traduction de $D P$ en langue étrangère (version italienne) a été réalisée par di Rosario et Borràs ${ }^{9}$. Les chercheuses ont mis en évidence l'évolution du rôle du traducteur à l'ère numérique : «Le traducteur ou la traductrice doit non seulement traduire des mots, mais aussi des images et des mouvements, et il ou elle peut être tenu d'avoir les compétences techniques pour le faire. » On entend ici par "compétences techniques " non seulement le degré de littératie numérique du traducteur (autrement dit, ses compétences informatiques), mais aussi une certaine familiarité avec la littérature numérique. Au cours des entretiens, di Rosario, Alonso et Marques ont précisé que DP était la première œuvre numérique qu'ils aient eu à traduire $^{10}$; Alonso notamment n'avait jusqu'alors jamais entendu parler de littérature numérique, faisant de la traduction de DP une expérience entièrement nouvelle pour elle.

8 Marques pose à ce sujet la question suivante: «Les œuvres de littérature numérique devraient-elles être traduites uniquement par des spécialistes de littérature numérique?» On peut répondre à la question de Marques en considérant qu'Alonso (version espagnole) n'est pas une spécialiste de littérature numérique et qu'elle est cependant parvenue à traduire l'œuvre en s'appuyant sur une correspondance par courrier électronique avec l'auteur. Cependant, le travail d'Alonso a principalement consisté à travailler sur le texte linguistique et sur la phonétique, plutôt qu'à partir du fichier code - comme ce fut le cas pour V. Bouchardon, di Rosario et Marques. Étant donné que Marques et di Rosario sont des chercheurs en littérature numérique, que $V$. Bouchardon enseigne l'anglais comme langue étrangère et qu'Alonso est traductrice, on peut se demander dans quelle mesure la trajectoire professionnelle du traducteur affecte la traduction du texte source numérique. Existe-t-il une littératie numérique propre au traducteur de littérature numérique?

L'enregistrement de voix (scènes 1, 2 et 4) au cours du processus de traduction est une caractéristique originale de $D P$. Un tel processus comprend la traduction de la transcription, la sélection d'une voix (une personne) pour l'interpréter, et enfin la lecture et l'enregistrement de la transcription traduite avant son envoi à l'auteur et au traducteur. Ainsi, au cours de son travail sur la version portugaise, Marques confie-t-il avoir dû «trouver trois personnes pour réciter le texte, et l'instrument pour les enregistrer ». De même di Rosario évoque-t-elle l'existence d'un élément technique essentiel à prendre en compte : «Traduire de la littérature numérique demande d'avoir à sa disposition différents instruments techniques d'enregistrement ${ }^{11}$. » Pour pouvoir s'enregistrer, V. Bouchardon a utilisé le même logiciel (Audacity) qu'elle emploie avec ses élèves, la dispensant d'avoir à apprendre à utiliser un nouvel outil. On constate ainsi que les différents degrés d'intégration des "compétences techniques" dans ces différentes méthodologies de traduction varient en fonction du parcours professionnel de chaque traducteur et de la collaboration entre auteur et traducteur. Un traducteur pourra en effet avoir besoin d'acquérir de nouvelles compétences en fonction des difficultés auxquelles il se trouve confronté. Ainsi, la traduction et l'enregistrement de 
contenu audio dans une autre langue ont-ils été présentés par Marques et di Rosario comme une étape cruciale de leur méthodologie de traduction, non seulement au regard des problèmes techniques rencontrés mais aussi des défis posés sur le plan de l'interprétation vocale, comme nous le verrons dans la partie «Son et sens ».

\section{Le visible et l'invisible}

10 Dans leur article « Renderings : Translating literary works in the digital age », Marecki

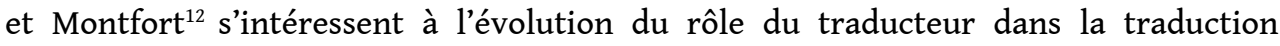
littéraire numérique par comparaison avec la traduction littéraire traditionnelle :

Dans la littérature traditionnelle, le traducteur est souvent invisible, simple personnage à l'arrière-plan qui peut être discrètement mentionné ou carrément oublié. Dans la littérature numérique, le traducteur acquiert une visibilité, il devient un ambassadeur de l'œuvre dont il explique souvent le mécanisme et le processus de traduction.

11 Les entretiens donnent à voir le traducteur comme une figure active et polyvalente dans son travail sur la littérature numérique. Il est en mesure de poser des questions, de discuter avec l'auteur d'aspects stylistiques, de développer sa propre méthodologie, d'apprendre de nouvelles compétences numériques (si nécessaire) et d'éprouver l'acte de traduction selon de nouveaux modes de communication esthétique et littéraire (par exemple en interagissant et en manipulant le texte numérique, par comparaison avec le texte imprimé). Il existe un dialogue, un échange d'idées et parfois une participation au processus de codage. Le traducteur est reconnu ( $c f$.les crédits de l'œuvre), peut prétendre à une voix et à un rôle dans le processus créatif.

12 La visibilité et l'invisibilité en jeu dans la traduction d'œuvres numériques peuvent être explorées sous un autre angle si nous revenons à l'idée d'un texte numérique invisible situé au-delà de l'écran et à celle de sa traduction linguistique visible. Comme l'explique di Rosario au sujet de sa propre expérience de traduction:

En un sens, j'estime que la traduction linguistique est « visible ", autrement dit que l'on peut passer à côté des connotations ou des nuances d'un mot sans pour autant avoir de difficultés à lire le texte, ne serait-ce que pour vérifier le terme original (le mot est là, immobile sur la page). Dans la littérature numérique, les choses se compliquent, car on peut évidemment traduire correctement le texte linguistique et en même temps perdre une partie du sens de l'œuvre - qui est aussi produit par d'autres mouvements, manipulations, etc.

13 Di Rosario insiste une fois de plus sur l'importance du rôle que jouent l'interaction et la manipulation de l'œuvre dans l'évaluation des effets esthétiques du texte linguistique par-delà l'écran. Au sein du processus de traduction semble ainsi se jouer une négociation entre le texte visible et invisible et son résultat esthétique. Marques ${ }^{13}$ revient quant à lui sur les notions de transparence et de translucidité, ainsi que sur les aspects intersémiotiques présents dans la traduction de textes numériques :

On notera que les idées de transparence et de translucidité des interfaces numériques, telles qu'elles sont promues par l'industrie numérique, peuvent être comparées à l'idée de transparence et de translucidité en traduction. Et notamment lorsqu'il s'agit de traduire quelque chose qui cesse d'être exclusivement discursif pour devenir multimodal et impliquer une perception multisensorielle.

14 Sans doute la traduction intersémiotique est-elle propre à la littérature numérique, où les sens de la vue, de l'ouïe et du toucher jouent un rôle fondamental pour la construction du sens de l'œuvre. Les remarques de Marques et di Rosario sont la preuve 
que leurs préoccupations en tant que traducteurs sont directement liées aux compétences qui sont déjà les leurs en tant que lecteurs de littérature numérique et chercheurs dans ce champ (comment lisons-nous la littérature numérique?). L'évocation des "figures de rhétorique numériques " chez di Rosario témoigne de la spécificité de cette dynamique cachée de la littérarité dans l'œuvre numérique, là où les remarques de Marques sur la complexité de la perception multisensorielle (cf.l'application mobile) témoignent des choix intersémiotiques (tels que les changements dans la temporalité, dans le contexte et dans la texture des ressources sémiotiques) que Bouchardon et lui ont dû effectuer au cours du processus de traduction collaborative.

\section{Traduire les dimensions propres à la littérature numérique}

\section{De Flash à JavaScript}

$D P$, comme de nombreuses autres œuvres littéraires numériques, est confronté à l'obsolescence des logiciels de programmation. Flash, le logiciel dans lequel les versions française, anglaise, italienne, espagnole et portugaise furent initialement programmées, a cessé de fonctionner en janvier 2021. Dans un effort pour adapter l'œuvre aux défis technologiques actuels, l'auteur a migré ces quatre versions vers JavaScript en 2018. Le processus traductif de pertes et de gains est ainsi reflété dans les différences qui apparaissent à l'écran entre les deux logiciels, certaines possibilités visuelles de la version Flash ayant disparu tandis que d'autres aspects esthétiques ont gagné en qualité avec JavaScript. Il importe de préciser que le présent article ne porte pas sur la réadaptation du texte linguistique entre une version anglaise Flash et une version anglaise JavaScript, par exemple. Cependant, nous reconnaissons qu'un travail de révision de la traduction est indispensable concernant les versions de l'œuvre déjà publiées et récemment reprogrammées en raison de l'obsolescence logicielle. 
Fig. 2

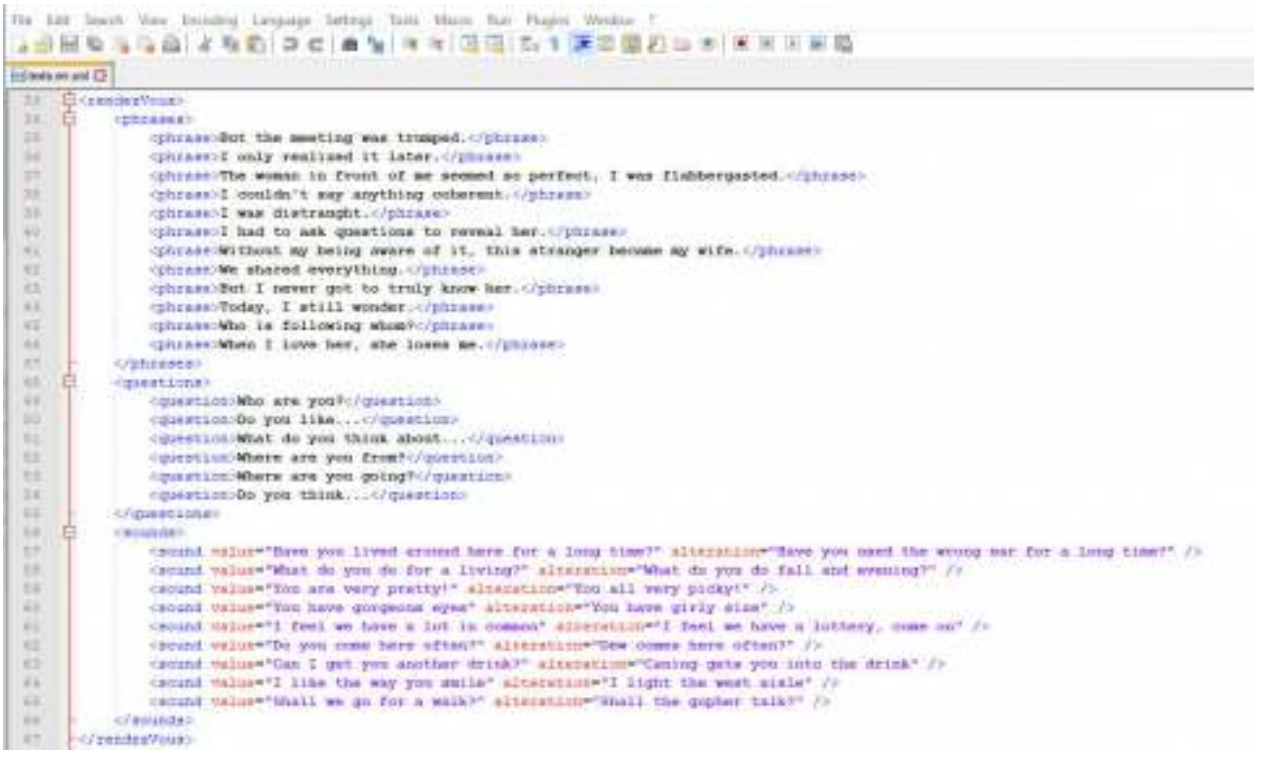

Exemple de code XML pour Loss of Grasp, scène 2 (version Flash, 2010).

Fig. 3

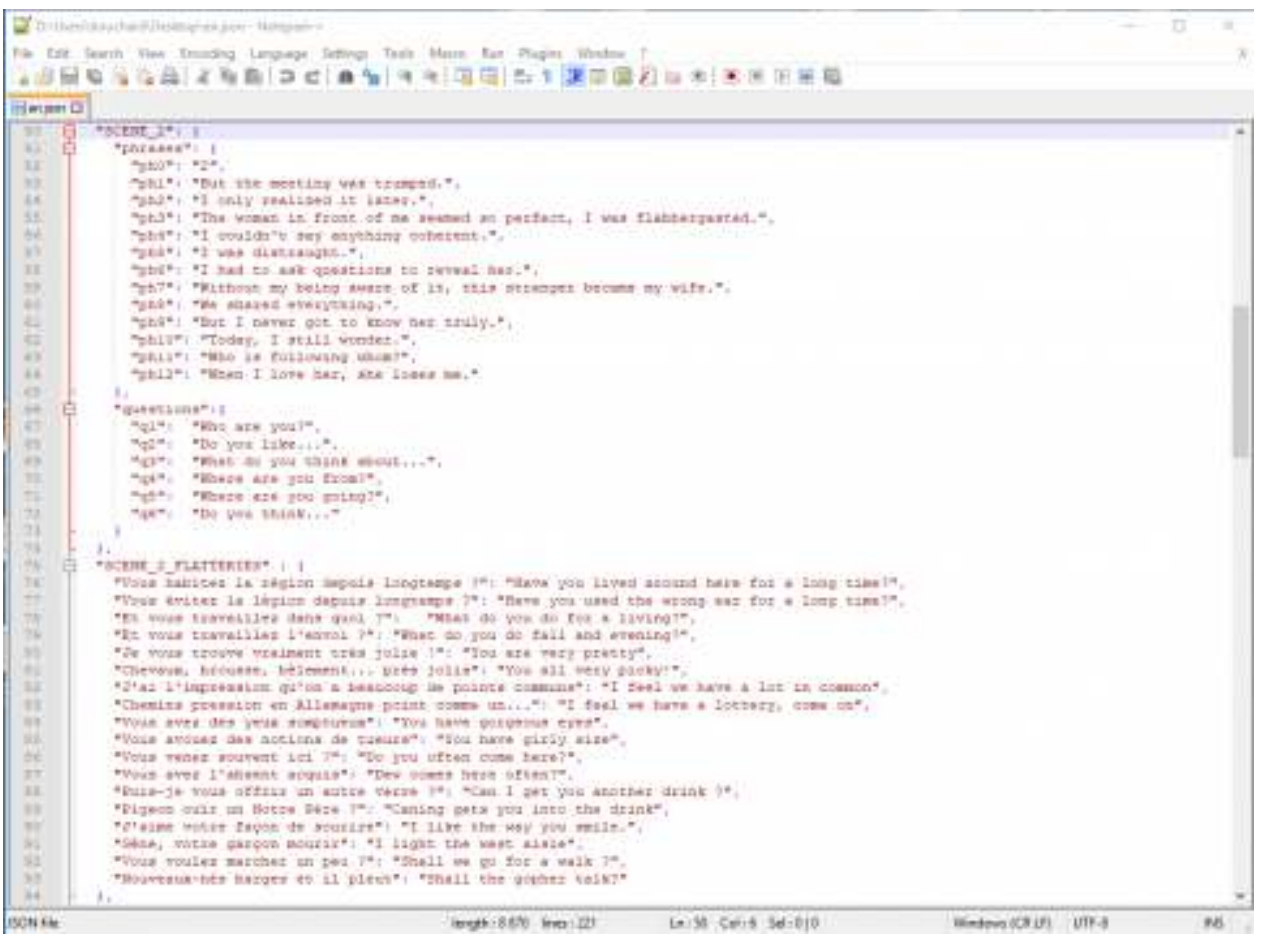

Exemple de fichier JSON pour Loss of Grasp, scène 2 (version mobile, 2018)

Dans ce cadre, on pourrait dire que $D P$ est le lieu d'une véritable opération de transcodage (de Flash à JavaScript) et de transmédialité (du PC à l'application pour smartphones et tablettes). Repenser les scènes en intégrant la dimension tactile (les smartphones n'ayant pas de curseur) implique un travail de transcréation complexe aussi bien pour l'auteur que pour les traducteurs et les traductrices. La traduction entre codes informatiques a ainsi des répercussions sur l'effet de glitch audiovisuel des 
versions JavaScript. La scène 1 (le passage tumultueux, la déprise absolue) n'est en effet pas rendue de la même manière en JavaScript, qu'il s'agisse de la version Web ou de l'application mobile, provoquant un ralentissement du déplacement sémiotique (figure 4). On citera entre autres exemples l'interaction avec la lettre d'amour/de rupture écrite par la femme du protagoniste dans la scène 3, où la lecture du texte fonctionne mieux à partir de mouvements de bas en haut (JavaScript) que de droite à gauche (Flash); et la scène 6 , où l'apparition de phrases individuelles paraît plus lente, moins saisissable, en JavaScript qu'en Flash. Il semblerait que le " principe compositionnel ${ }^{14}$ " de l'œuvre ne s'exprime pas de la même manière en Flash et en JavaScript.

Fig. 4

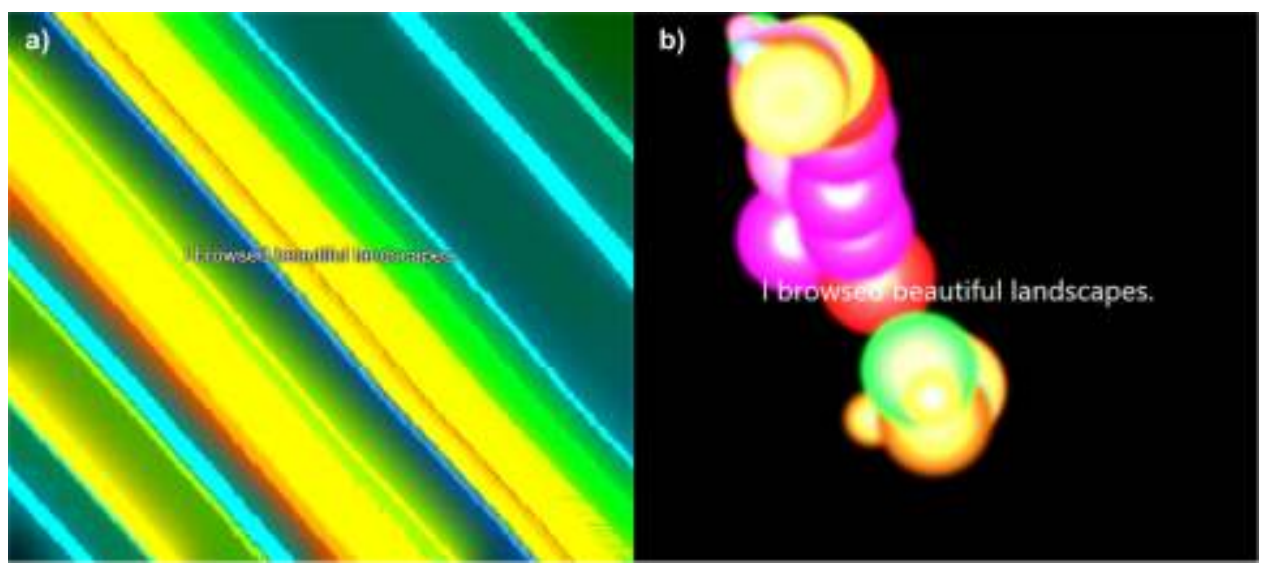

a) Extrait de la scène 1 (Flash) ; b) Extrait de la scène 1 (JavaScript, version Web).

\section{Formes et gestes sémiotiques}

17 L'interrelation entre ressources sémiotiques (mots, images, sons, gestes, etc.) joue un rôle clé dans les pratiques de traduction littéraire numérique. La traduction de certaines œuvres littéraires numériques permet-elle réellement de parvenir à une cohérence intersémiotique? Par exemple, lorsque nous recréons des effets littéraires similaires (des figures de style et de rhétorique) dans les différentes versions de l'œuvre? Dans DP, ces effets littéraires et rhétoriques sont rendus possibles grâce aux «figures de manipulation» (gestuelle). Les figures résident dans le décalage entre les attentes du lecteur dans sa manipulation du texte et le résultat à l'écran ${ }^{15}$. La traductibilité de l'effet littéraire des figures de manipulation semble dès lors exiger un travail de traduction intersémiotique scrupuleux dans toutes les langues cibles (anglais, italien, espagnol, portugais).

Prenons pour exemple la scène 2 (le rendez-vous au cours duquel le protagoniste fait la rencontre de sa future femme), où un point d'interrogation apparaît à l'écran dans l'attente que le lecteur en explore la fonctionnalité. Après quelques secondes, le lecteur comprend qu'il doit cliquer sur le point d'interrogation pour qu'une série de questions apparaisse de façon aléatoire à l'écran : "Qui êtes-vous? ", «Vous aimez...? ", «Vous pensez quoi de...? ", «D'où venez-vous? ", « Où allez-vous? " "Que pensez-vous... ?» (figure 5a). Dans la lignée de Portela, Pold et Mencía ${ }^{16}$, on notera qu'il s'agit d'un moment clé pour la traduction de l'œuvre à différents niveaux : 1) translinguistique, les traducteurs et les traductrices ayant dû traduire les différentes versions du texte 
linguistique à partir de la langue source dans les différentes langues cibles (figure $5 b, 5 c$ et $5 \mathrm{~d})$; 2) transcodage, l'auteur ayant eu à reproduire cet effet dans les six nouvelles versions JavaScript, ainsi que dans l'application mobile (migration entre plateformes); 3) transmédialité, les traducteurs et les traductrices et l'auteur ayant dû intégrer certains modalités sémiotiques dans l'application mobile (notamment une narration visuelle multilingue pour le personnage de la femme) ; 4) transcréation, l'auteur et les traducteurs et les traductrices ayant dû reproduire la littérarité et la traductibilité des "figures de manipulation» (interaction et manipulation) qui dévoilent l'image du personnage de la femme. Le travail de traduction a ainsi consisté à créer une harmonie entre l'interaction, la manipulation et les textes linguistiques dans différentes langues qui construisent la même image. Le but n'est pas seulement de saisir le sens des éléments d'expression esthétique, mais de rechercher des motifs littéraires.

Fig. 5
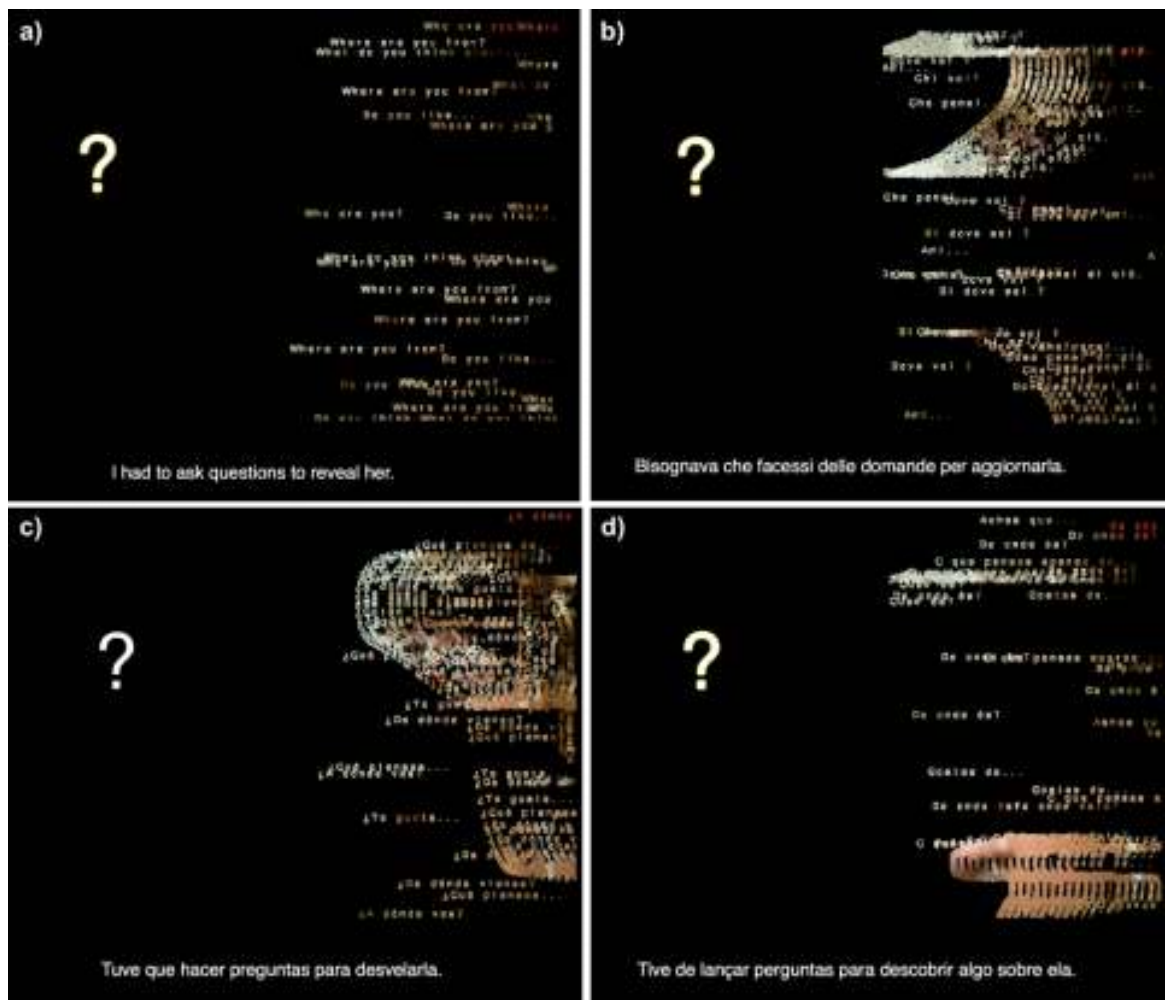

Déprise, version Flash, scène 2, langues cibles a) anglais, b) italien, c) espagnol, d) portugais.

\section{Son et sens}

DP est une création polyphonique. La traduction des différentes voix occupe une place essentielle dans le travail de traduction. La voix du narrateur, la voix d'une standardiste et d'un adolescent se font entendre au fil des scènes. Ces voix forment des conversations à sens unique, empruntant différents rythmes, qui engendrent une atmosphère d'hétérophonie numérique.

Dans la scène 2 (le rendez-vous du protagoniste avec sa future femme), l'auteur joue sur ce qu'il appelle des «altérations ", ou des malentendus entre la voix entendue et ce qui apparaît à l'écran. On notera que ces malentendus (mal entendus) ont été produits à 
l'origine en utilisant un logiciel de reconnaissance vocale qui ne retranscrivait pas les propos de façon fidèle.

a. Vous habitez la région depuis longtemps? (Vous évitez la légion depuis longtemps)

b. Et vous travaillez dans quoi ? (Et vous travaillez l'envoi ?)

c. J'ai l'impression qu'on a beaucoup de point communs (J'ai la pression et la pinte en commun)

d. Je vous trouve vraiment très jolie! (Chevaux, brousse, bêlement... prés jolis)

e. J'aime votre façon de sourire (Gêne, votre face a des soupirs)

f. Vous voulez marcher un peu ? (Nouveau-nés barges et il pleut)

21 Hormis V. Bouchardon, aucun des traducteurs et des traductrices ne savait qu'un logiciel de reconnaissance vocale avait été à l'origine de ces altérations, mais ils avaient tous conscience d'un jeu phonétique. Le défi stylistique fut vécu de manière différente par chacun d'entre eux, comme l'explique di Rosario :

Une autre difficulté tenait à ce que Bouchardon appelle les «altérations »: d'ailleurs, dans la scène 2, certaines phrases ont été changées. J'ai laissé certains sens, surtout dans les phrases principales, mais j'ai joué sur la sonorité des mots dans l'« altération ", comme Bouchardon l'avait fait dans la version française, de sorte que le sens de certaines phrases est tout autre.

Alonso fait les observations suivantes :

Quand j'avais eu à traduire Déprise, j'avais travaillé les altérations dans le but d'obtenir les mêmes effets de surprise qu'en français. Pour cela, j'avais joué assez librement sur la phonétique. Je considère que cet aspect (la recréation des rythmes et des sonorités) constitue le principal défi de toute traduction. Cela me semble revêtir une importance majeure dans le cas d'une œuvre numérique, faisant directement appel à tous les sens du récepteur ${ }^{17}$.

Quant à Marques, il décrit ainsi sa propre expérience :

Il m'a paru particulièrement compliqué de «transcoder » en portugais cet humour si français qui parcourt toutes les fictions interactives de Serge. C'est notamment le cas pour les transferts culturels, avec des expressions idiomatiques épineuses et la difficulté supplémentaire de la traduction des homophones.

Pour V. Bouchardon, l'expérience fut légèrement différente. La traduction de cette partie est le résultat d'une discussion continue avec son mari (l'auteur). En outre, en tant qu'enseignante d'anglais langue étrangère, V. Bouchardon a l'habitude d'entendre ses élèves confondre les sons et les mots en anglais. C'est sur cette expérience d'enseignante qu'elle s'est appuyée pour rendre les « altérations ».

Exemples de traduction, scène 2

Français (original) :

"J'aime votre façon de sourire "

(Gêne, votre face a des soupirs);

Anglais :

«I like the way you smile »

(I light the west aisle) ;

Italien :

« Mi piace il tuo modo di sorridere »

(So il tuo ruolo nell'uccidere);

Espagnol :

« Me encanta tu sonrisa »

(Andar por la cornisa) ;

Portugais :

"Gosto da maneira como sorris "

(Gosto da bandeira como só ris) 
Traduire la dichotomie entre le sens et le son est l'une des grandes difficultés de la scène 2. L'imagination et les stratégies stylistiques du traducteur témoignent d'un véritable processus de transcréation, la version originale étant le fruit d'un logiciel de reconnaissance vocale. L'exercice linguistique et phonétique n'a pas manqué de faire naître certaines interrogations : comment traduire l'humour culturel dans la littérature numérique? Comment différentes cultures interprètent-elles $D P$ ? Le fait que $D P$ continue à être traduit dans différentes langues lui confère une dimension de "traduction prismatique ${ }^{18}$ ». D'une part, les voix de la scène 2 appartiennent à différents contextes culturels (France, Espagne, Italie, Portugal) et différents individus ; d'autre part, des éléments culturels aléatoires ont été utilisés dans les traductions pour répondre à chaque contexte particulier. Ces traductions donnent en outre à voir l'inextricable enchevêtrement $d u$ multilinguisme et du multiculturalisme comme produit et défi de la traduction.

\section{Futurs axes de recherche}

\section{Le rôle de la traduction indirecte}

Les versions française (originale) et anglaise de DP sont parues en même temps, en 2010. Au cours de nos entretiens, les traducteurs et les traductrices ont indiqué que la langue source avait alterné, pour certains d'entre eux, entre le français et l'anglais. Il faut alors considérer deux aspects: 1) la connaissance d'autres langues par le traducteur ; 2) les traductions existantes au moment d'une nouvelle version. Nous savons avec certitude que le texte source employé par V. Bouchardon est le texte français (2010). Di Rosario (2011) et Alonso (2013) ont également travaillé à partir de la version française (2010), tout en gardant la possibilité d'effectuer des comparaisons esthétiques et linguistiques avec la version anglaise (2010). Enfin, le texte source utilisé par Marques (2016) était le texte anglais, le traducteur ayant par ailleurs les compétences linguistiques suffisantes pour lire la version française et réaliser des comparaisons à partir des versions espagnole et italienne. Les interrelations entre langues source et cible permettent de parler d'un processus de traduction indirecte en littérature numérique (la traduction d'une traduction ${ }^{19}$ ).

Comme nous pouvons le constater, la version anglaise de V. Bouchardon a servi de référent, l'anglais jouant le rôle de langue de référence et de rencontre entre traducteurs (lingua franca). Dès lors, nous pouvons nous demander dans quelle mesure la traduction anglaise de V. Bouchardon a influencé les autres versions de DP. Lequel serait considéré comme le texte source : Déprise (2010), Loss of Grasp (2010), ou bien les deux? Quelle est la visibilité et l'incidence littéraire des traductions indirectes dans les futures versions de DP? Dans une étude critique ultérieure, nous pourrons ainsi explorer les possibilités méthodologiques de la traduction indirecte dans la littérature numérique (emprunts, comparaisons, dissemblances), prenant pour étude de cas les six nouvelles versions de DP (arabe, chinoise, allemande, hongroise, polonaise et russe).

\section{La dimension culturelle}

DP comprend des passages qui se rapportent strictement à la culture occidentale. Ainsi, dans la scène 3 , de la lecture de gauche à droite de la lettre d'amour/de rupture, par 
comparaison avec une lecture de droite à gauche dans la version arabe; dans la scène 3 également, de l'utilisation de Carmen de George Bizet en musique de fond; dans la scène 4 , du concept de "héros " convoqué par le fils du protagoniste; dans la scène 4 à nouveau, de l'emploi du mot "zoïle» (un critique grec); etc. L'adaptation d'effets esthétiques et poétiques de ce type aux différentes versions soulève certaines questions : comment remplacer ou compenser de tels aspects culturels dans la culture cible ? Comment aborder les transferts culturels dans la traduction d'œuvres littéraires numériques? Pour le cas de la lettre d'amour/de rupture dans la version arabe, on constate ainsi que le double sens et l'effet visuel du texte linguistique répondent bien au sens de l'œuvre, mais ne sont pas culturellement cohérents avec la langue cible. Quelque chose de semblable se produit avec le Carmen de Bizet, puisque l'opéra joue un rôle essentiel dans la construction de l'environnement littéraire de la scène au moment de la lecture de la lettre, mais pourrait ne pas convenir aux différents contextes de traduction; de même pour la traduction du mot (du personnage) de «zoïle » dans les différentes langues.

Dans son article « Digital cultures : A view from French studies and literature ${ }^{20}$ », Erika Fülöp évoque le désir de « saper le stéréotype d'une culture mondiale homogène à l'ère numérique ", rappelant que la littérature numérique préserve les traces de cultures prénumériques. Le raisonnement de Fülöp est étroitement lié à la traduction d'œuvres de littérature numérique contemporaines. Le rôle du traducteur (en anglais, par exemple) devrait-il être de diluer les références culturelles pour qu'un public anglophone puisse s'identifier à l'œuvre, ou au contraire de reproduire la moindre trace de spécificité culturelle afin d'accentuer la diversité culturelle des œuvres littéraires numériques aux dépens de leur dimension internationale ${ }^{21}$ ? Dès lors, la diversité culturelle s'exprime-t-elle exclusivement à travers la dimension linguistique de la littérature numérique ? Et quid du rapport et de l'équilibre entre esthétique numérique et transferts culturels?

\section{Le numérique : une mémoire réinventée}

30 Si nous abordons les traductions d'œuvres littéraires numériques comme des archives d'éléments culturels et technologiques - le logiciel informatique qui encode les modes d'expression d'une période informatique donnée (l'obsolescence de Flash et, peut-être à l'avenir, de JavaScript), la traduction culturelle d'expressions idiomatiques, les adaptations culturelles des beaux-arts - il est possible d'établir un lien entre traduction et préservation. Or, la valeur ajoutée de la technologie numérique ne se trouve pas là où l'on pourrait s'y attendre. Le support numérique n'est pas un mode de préservation naturel, mais au contraire un enfer pour la préservation. La technologie numérique nous fait néanmoins pénétrer dans un nouvel univers qui est un univers de mémoire non pas archivée, mais réinventée $e^{22}$.

31 D'un point de vue anthropologique, ce modèle de la mémoire est plus riche et plus authentique que le modèle du support papier, fondé sur l'archivage (le livre que l'on range sur une étagère, pareil au souvenir que l'on range dans une case de son cerveau). En effet, les sciences cognitives nous enseignent que la mémoire ne fonctionne pas sur le modèle de l'archivage et de la conservation. Préserver, c'est donc constamment réinventer le contenu - à l'instar de ce que fait la traduction. L'enjeu est d'obtenir une invention fidèle et juste, une reconstruction dans laquelle les changements sont 
explicites et explicités. De même, la traduction donne à voir dans l'ère numérique le passage d'un modèle de la mémoire archivée à celui d'une mémoire continuellement réinventée. Dans cette perspective, compte tenu de l'interrelation entre formes intersémiotiques (mots, images, sons, gestes) au sein de la littérature numérique et tout en gardant à l'esprit la proposition de Reynold pour une "traduction prismatique ${ }^{23}$ ", les œuvres littéraires numériques peuvent être envisagées comme un bon laboratoire pour expérimenter avec la traduction à l'ère numérique.

\section{Conclusion}

Cette étude de cas a été conçue pour étudier les enjeux de la traduction d'une création de littérature numérique dans différentes langues. Les entretiens avec les traducteurs et traductrices ont permis d'obtenir une meilleure vue d'ensemble des différents processus et méthodologies employés, notamment lorsque plusieurs langues cibles sont en jeu. Nous avons décrit comment le processus de traduction a donné lieu à une tension créative entre médias, formes sémiotiques, écriture programmée, expérience esthétique et aspects culturels. Cette étude de cas nous a aidé à mieux comprendre comment ces différents éléments devaient s'adapter non seulement à une culture et langue cibles, mais également à un code cible.

Les quatre dimensions de la traduction de la littérature numérique ${ }^{24}$ ont été relevés dans le processus de traduction. Au niveau translinguistique, le recours à la traduction indirecte s'est révélé être un instrument de communication entre les traducteurs. Au niveau de la transmédialité et du transcodage, les traducteurs et traductrices ont été confrontés au défi de traduire le texte linguistique comme les autres formes sémiotiques, mais également dans certains cas de les adapter à un autre langage de programmation ou une autre plate-forme. Enfin, au niveau de la transcréation, nous avons montré que la collaboration entre l'auteur et les traducteurs et traductrices était essentielle pour reproduire la littérarité et les éléments esthétiques de l'œuvre. Plus généralement, la diversité croissante des genres et des dispositifs de la littérature numérique déterminera non seulement l'évolution des méthodologies de traduction, mais contribuera aussi à redéfinir les axes de recherche du domaine en tant que tel.

\section{BIBLIOGRAPHIE}

Bouchardon, Serge et Bachimont, Bruno, « Preservation of digital literature : From stored memory to reinvented memory ", Cibertextualidades $n^{\circ}$ 5, 2013, p. 184-202. [En ligne] http:// cibertextualidades.ufp.edu.pt/numero-5-2013/electronic-publishing-models-for-experimentalliterature [consulté le 23 juillet 2020].

Bouchardon, Serge, « Figures of gestural manipulation in digital fictions », in Bell, Alice, Ensslin, Astrid et Rustad, Hans (dir.), Analyzing Digital Fiction, Londres, Routledge, 2014, p. 159-175. 
Bouchardon, Serge, « Mind the gap! 10 gaps for digital literature ? », Electronic Book Review, 2019. [En ligne] http://electronicbookreview.com/essay/mind-the-gap-10-gaps-for-digital-literature/ [consulté le 23 juillet 2020].

Cayley, John, « Translation as process », Amodern, $n^{\circ}$ 8, Christine Mitchell et Rita Raley (dir.), « Translation-Machination », 2018. [En ligne] http://amodern.net/issues/amodern-8-translationmachination/ [consulté le 23 juillet 2020].

Di Rosario, Giovanna et Borràs, Laura, «Translating digital literature : Two experiences and a reflection », Texto Digital, vol. 8, n 1, 2012, p. 138-162. [En ligne] https://doi.org/ 10.5007/1807-9288.2012v8n1p138 [consulté le 23 juillet 2020].

Fülöp, Erika, « Digital cultures : A view from French studies and literature », Explorations in Media Ecology, vol. 17, n 3, 2018, p. 271-277. [En ligne] https://doi.org/10.1386/eme.17.3.271_1 [consulté le 23 juillet 2020].

Gambier, Yves, «La retraduction, retour et détour », Meta. Journal des traducteurs/Meta. Translators' Journal, vol. 39, $\mathrm{n}^{\circ}$ 3, 1984, p. 413-417. [En ligne] https://doi.org/10.7202/002799ar [consulté le 23 juillet 2020].

Marecki, Piotr et Montfort, Nick, « Renderings : Translating literary works in the digital age ", Digital Scholarship in the Humanities, vol. 32, n 1, 2017, p. 84-91. [En ligne] https://doi.org/10.1093/ llc/fqx010 [consulté le 23 juillet 2020].

Marques, Diogo, Reading Digits. Haptic Reading Processes in the Experience of Digital Literary Works, thèse de doctorat, Portugal, Universidade de Coimbra, 2018. [En ligne] https:// estudogeral.sib.uc.pt/handle/10316/81171?mode=simple [consulté le 23 juillet 2020].

Portela, Manuel, Pold, Søren et Mencía, María, « Electronic literature translation : Translation as process, experience and mediation », Electronic Book Review, 2018. [En ligne] http:// electronicbookreview.com/essay/electronic-literature-translation-translation-as-processexperience-and-mediation/ [consulté le 23 juillet 2020].

Reynolds, Matthew, Translation. A Very Short Introduction, Oxford, Oxford University Press, 2016.

\section{NOTES}

1. Serge Bouchardon et Vincent Volckaert, Déprise, 2010. [En ligne] http://deprise.fr ou http:// lossofgrasp.com [consultés le $1^{\mathrm{er}}$ avril 2021].

2. Yves Gambier, «La retraduction, retour et détour », Meta. Journal des traducteurs/Meta. Translators' Journal, vol. 39, n³, 1984, p. 413-417. [En ligne] https://doi.org/10.7202/002799ar [consulté le 23 juillet 2020].

3. Manuel Portela, Søren Pold et María Mencía, « Electronic literature translation : Translation as process, experience and mediation ", Electronic Book Review, 2018. [En ligne] http:// electronicbookreview.com/essay/electronic-literature-translation-translation-as-processexperience-and-mediation/ [consulté le 23 juillet 2020].

4. Serge Bouchardon et Vincent Volckaert, Déprise, 2010. [En ligne] http://deprise.fr ou http:// lossofgrasp.com [consultés le $1^{\mathrm{er}}$ avril 2021].

5. Serge Bouchardon, Léonard Dumas, Vincent Volckaert, Hervé Zénouda, Giovanna di Rosario (traductrice) et Valérie Bouchardon (traductrice), Opacité, 2012. [En ligne] http://i-trace.fr/ opacite/ [consulté le $1^{\mathrm{er}}$ avril 2021].

6. Serge Bouchardon, Clément Routier, Agathe Guillemot, Antoine Aufrechter, Elsa Chaudet et Vincent Volckaert, Détrace, 2016. [En ligne] https://i-trace.fr/detrace/ [consulté le $1^{\mathrm{er}}$ avril 2021]. 
7. Maria Engberg, Talan Memmott et David Prater (dir.), Anthology of European Electronic Literature, 2012. [En ligne] https://anthology.elmcip.net/ [consulté le 29 mars 2021].

8. Pour d'avantage d'informations à ce sujet, voir [en ligne] http://deprise.fr ou http:// lossofgrasp.com. Pour une capture vidéo des interactions, voir [en ligne] https://youtu.be/ nd6_b158qOs [consulté le 29 mars 2021].

9. Giovanna Di Rosario et Laura Borràs, «Translating digital literature : Two experiences and a reflection ", Texto Digital, vol. 8, n 1, 2012, p. 138-162.

10. V. Bouchardon avait déjà traduit d'autres œuvres du même auteur: Les Douze Travaux de l'internaute (2008) et Toucher (2009) ; di Rosario et Marques traduiront plus tard Opacité (2012) et Détrace (2016).

11. On notera que V. Bouchardon et Marques ont utilisé leur propre voix dans les versions anglaise et portugaise. Nous avons là un exemple de transcréation traductive, les traducteurs et les traductrices interprétant leur traduction linguistique en enregistrant leur voix et en l'intégrant à l'œuvre numérique. Cet élément vient étayer l'idée du traducteur comme collaborateur-médiateur présent non seulement à travers ses mots (texte linguistique) mais sa voix (audio).

12. Piotr Marecki et Nick Montfort, « Renderings : Translating literary works in the digital age », Digital Scholarship in the Humanities, $\mathrm{n}^{\circ} 32$, suppl. 1, 2017, p. 84-91.

13. Diogo Marques, Reading Digits. Haptic Reading Processes in the Experience of Digital Literary Works, thèse de doctorat, Portugal, Universidade de Coimbra, 2018. [En ligne] https:// estudogeral.sib.uc.pt/handle/10316/81171?mode=simple [consulté le 23 juillet 2020].

14. John Cayley, "Translation as process ", Amodern, $n^{\circ} 8$, Christine Mitchell et Rita Raley (dir.), « Translation-Machination », 2018. [En ligne] http://amodern.net/issues/amodern-8-translationmachination/ [consulté le 23 juillet 2020].

15. Serge Bouchardon, « Figures of gestural manipulation in digital fictions », in Alice Bell, Astrid Ensslin et Hans Rustad (dir.), Analyzing Digital Fiction, Londres, Routledge, 2014, p. 159-175.

16. Manuel Portela, Søren Pold et María Mencía, « Electronic literature translation : Translation as process, experience and mediation", Electronic Book Review, 2018. [En ligne] http:// electronicbookreview.com/essay/electronic-literature-translation-translation-as-processexperience-and-mediation/ [consulté le 23 juillet 2020].

17. On notera que la traductrice espagnole (Alonso) communiquait avec l'auteur en français.

18. Matthew Reynolds, Translation. A Very Short Introduction, Oxford, Oxford University Press, 2016.

19. Yves Gambier, "La retraduction, retour et détour", Meta. Journal des traducteurs/Meta. Translators' Journal, vol. 39, n³, 1984, p. 413-417. [En ligne] https://doi.org/10.7202/002799ar [consulté le 23 juillet 2020].

20. Erika Fülöp, «Digital cultures: A view from French studies and literature », Explorations in Media Ecology, vol.17, n 3, 2018, p. 271-277. [En ligne] https://doi.org/10.1386/eme.17.3.271_1 [consulté le 23 juillet 2020].

21. Serge Bouchardon, "Mind the gap! 10 gaps for digital literature?", Electronic Book Review, 2019. [En ligne] http://electronicbookreview.com/essay/mind-the-gap-10-gaps-for-digitalliterature/ [consulté le 23 juillet 2020].

22. Serge Bouchardon et Bruno Bachimont, «Preservation of digital literature : From stored memory to reinvented memory ", Cibertextualidades, $n^{\circ} 5,2013$, p. 184-202. [En ligne] http:// cibertextualidades.ufp.edu.pt/numero-5-2013/electronic-publishing-models-for-experimentalliterature [consulté le 23 juillet 2020].

23. Matthew Reynolds, Translation. A Very Short Introduction, Oxford, Oxford University Press, 2016.

24. Portela, Manuel, Pold, Søren et Mencía, María, « Electronic literature translation : Translation as process, experience and mediation", Electronic Book Review, 2018. [En ligne] http:// 
electronicbookreview.com/essay/electronic-literature-translation-translation-as-processexperience-and-mediation/ [consulté le 23 juillet 2020].

\section{RÉSUMÉS}

Déprise est une création de littérature numérique mise en ligne en 2010 (http://deprise.fr ou http://lossofgrasp.com). Progressivement, ce récit interactif a été traduit en anglais (2010), italien (2011), espagnol (2013) et portugais (2016), puis plus récemment en arabe, chinois, allemand, hongrois, polonais et russe (2020). Chaque traduction a conduit à un processus interculturel et transcréatif (Portela, Pold \& Mencia, 2018) entre les traducteurs et les traductrices et l'auteur. À travers des entretiens, nous avons demandé aux traducteurs et aux traductrices de Déprise en anglais, italien, espagnol et portugais leurs impressions sur le processus de traduction. Dans cet article, nous nous appuyons sur les échanges écrits avec les traducteurs et les traductrices pour questionner les modes de collaboration entre auteur et traducteur et l'importance de la traduction des dimensions propres à la littérature numérique. Nous évoquerons également plusieurs axes de recherche futurs sur la traduction de littérature numérique : le rôle de la traduction indirecte (Gambier, 1994), la dimension culturelle des œuvres et la traduction comme mémoire réinventée.

\section{INDEX}

Mots-clés : littérature numérique, traduction, multilinguisme, transcréation

\section{AUTEURS}

\section{SERGE BOUCHARDON}

Serge Bouchardon (http://www.utc.fr/ bouchard/), agrégé de lettres modernes, est actuellement professeur en sciences de l'information et de la communication à l'université de technologie de Compiègne. Son travail de recherche porte sur les écritures numériques, en particulier sur la littérature numérique. Parmi ses publications : La Valeur heuristique de la littérature numérique (Hermann, Paris, «Cultures numériques », 2014). En tant qu'auteur de littérature numérique (http://www.sergebouchardon.com/), il s'intéresse notamment au rôle du geste dans l'écriture interactive. Ses créations ont été présentées en diverses occasions en Europe, en Amérique, en Afrique et au Moyen-Orient. Elles ont fait l'objet de publications dans des revues en ligne (bleuOrange, Hyperrhiz, SpringGun, The New River...).

\section{NOHELIA MEZA}

Nohelia Meza a obtenu son doctorat de traduction et sciences du langage à l'université Pompeu Fabra de Barcelone en 2017. Elle développe actuellement son projet de recherche postdoctorale : «Vers une rhétorique numérique des œuvres latino-américaines de littérature électronique » à l'université de Leeds, Royaume-Uni (CONACYT, 2018-2020). Ses intérêts de recherche englobent les études culturelles latino-américaines, la rhétorique numérique, la traductologie, et la 
littérature comparée. Elle est membre du Réseau latino-américain de littérature électronique (litElat) et du Centre pour les cinémas du monde et la culture numérique (Université de Leeds). Elle est collaboratrice du groupe d'édition (projet de littérature électronique) au Centre pour la culture numérique à Mexico. 